\title{
The New Aspects of Current Language Survey in Rural China
}

\author{
Qijun Guo \\ College of Chinese Language and Culture \\ Jinan University \\ Guangzhou, China 510610
}

\begin{abstract}
Based on the existing research results in related fields, this paper briefly introduces the new changes in rural language life at present. Furthermore, this paper focuses on several new aspects of the current rural language survey, namely, the language life survey of empty nesters, left-behind children, migrant children and returning migrant workers as well as the survey of primary orality in rural areas. It is of practical and positive significance to carry out rural language survey for understanding the language life of special groups in rural areas and preserving the original rural language and culture.
\end{abstract}

\section{Keywords—rural language; survey; new aspects}

\section{INTRODUCTION}

As the name suggests, language survey investigates the language and language life of a particular region, community or group in order to understand the language use and language life of that region, community, or group. Sociolinguistics needs to study language in combination with the social environment of language. The paradigms of language research as well as the points of attention will be different in various language use environments. There are language surveys in different countries as well as in different areas within the same country. Besides, there are both urban language surveys and rural language surveys. From the perspective of user groups, there are also language surveys formed by different gender, age and other natural characteristics or by different occupations and other social characteristics, such as the language survey of migrant workers in cities.

Judging from the scope of investigation, the current language surveys in China are mainly in the following aspects: first, language surveys at the national level, mainly including language policy, language planning, national language ability and other aspects; second, language surveys at the city-level. At present, language survey at the city-level has formed a relatively fixed research paradigm, theoretical basis and investigation methods. And a lot of relevant research results have been found. These surveys mainly focus on the use of urban dialects and Mandarin, the language use and language attitudes of urban residents, the language use and language adaptation of specific social groups (such as migrant workers and ethnic minorities) in the process of urbanization, and the language status as well as the maintenance of urban dialects of special speech communities in cities (such as new industrial zones, science islands, etc.); third, language surveys at the rural level. The investigation of rural language usage began at the perspective of dialects and folklore. Dialect investigators usually use Fangyan Diaocha Zibiao and the Handbook for Grammatical Investigation and Research as the tools to collect dialect data by means of pronunciators' reading, inducing pronunciators to come up with grammatical structures, sentences or recording the most natural dialect materials. On this basis, it summarizes the local dialects and features, such as the phonetic characteristics, vocabulary use, grammatical characteristics, dialect that reflects the folk culture and so on.

In the 1950s and 1960s, rural literacy education was carried out on the education situation and literacy situation of rural villagers. In the $70 \mathrm{~s}$ and $80 \mathrm{~s}$, the Speak Mandarin Campaign was conducted to investigate the use of rura Mandarin. Since the beginning of the new century, some scholars have re-investigated the literacy situation of rural residents and the use of Mandarin. For example, Chen Weilin and Chen Xingyan ${ }^{1}$ investigated the popularization of Mandarin in rural middle and primary schools in southern Henan; Zhao Xiaogang ${ }^{2}$ investigated the views on common language and characters and the use of Mandarin in rural areas of Gansu, Qinghai and Ningxia.

In recent years, the Re-discussion of the relationship between dialects and Mandarin has aroused the attention of sociolinguists to the variation of rural dialects, which has been studied by $\mathrm{Fu}$ Yirong ${ }^{3}$, Wang Qing, Wu Xiaojun ${ }^{4}$ and so on. Compared with urban language survey, rural language survey is slightly inadequate in terms of investigation

Chen Weilin, Chen Xingyan. The Investigation and Reflection on the Situation of Tuipu in the Rural Primary and Middle Schools, Applied Linguistics, 2004(4)

2 Zhao Xiaogang. A Study on the Perception of General Language and Writing of People in Rural Gansu, Applied Linguistics, 2007(4)

FU Yirong. Migration and the Popularization of Putonghua: A Case Study of Migrant Workers from Fucun Village of Wuwei, Anhui Province, Applied Linguistics, 2010 (2).

WANG Qing \& Wu Xiaojun. New Research Perspective of Common Language and Dialect Variation - An Empirical Study on Pronunciation Variations of Sichuan Returned Migrant Workers, Journal of Xihua University (Philosophy \& Social Sciences), 2012 (6). 
intensity, scope, and theoretical basis and investigation methods. This paper draws on the urban language survey and refers to the rural language survey from the perspective of sociolinguistics as the rural language survey. Starting from the new changes in the current rural language life, this paper mainly discusses several new aspects of the current rural language survey, with a view to arousing the attention of the academic community to these aspects of the survey.

\section{NEW Changes In RURAL LANGUAGE LiFE}

\section{A. Changes in Blood Relationship and Geopolitical Relations and Rural Language Life}

The traditional rural area is a social network composed of blood and geopolitical relations. From the perspective of language acquisition, language acquisition in childhood mainly comes from oral transmission of family networks formed by parents and their families. And then people have the opportunity to access school language education. Some members of rural society may not receive school education for life. From the perspective of language use, one is the family network based on blood relationship, and the other is the village based on geographical relationship. In this context, the language use of rural residents is limited to interpersonal communication. As Fei Xiaotong ${ }^{5}$ said, the immobility of rural society in China determines that rural information in China is realized according to the information dissemination logic of acquaintances. In this mode of communication, the dissemination of information has a specific channel and mode. Limited by their radius of survival and radius of life, the information exchange of rural personnel is the information exchange between acquaintances and acquaintances. Language and writing sometimes prevent the exchange of information between people in rural society, where there is no news, "all cultures can be taught individually."

In the modern sense, the countryside has changed from a natural village formed by traditional geographical relationship to an administrative village managed by administrative divisions. Li Hongyan ${ }^{6}$ calls the natural village based on geographical relationship "acquaintance society" and the administrative village based on administrative division "semi acquaintance society". With the development of society and the convenience of transportation, the life radius of rural people is further expanded, and the channels of obtaining information tend to be diversified. The face-to-face communication mode based on "acquaintance-acquaintance" is changing. In the channels for farmers to obtain information, there is not only "acquaintances-strangers" interpersonal communication, but also mass communication based on television, telephone and radio.

Fei Xiaotong: "From the Soil: The Foundations of Chinese Society", Peking University press, 2005. press, 2014.

\section{B. Floating Population in Rural Areas - the New Influence of College Students and Migrant Workers on Rural Language Life}

On the one hand, more and more college students go out to study while migrant workers go out to work in agriculture. They maintain a "semi-detached" and "semi-related" relationship with the countryside. The relationship between these groups and the original countryside has gradually become defamiliarized and marginalized. And the familiarity and awareness of the villagers in the countryside has gradually decreased. In addition, the defamiliarization of the new generation of rural workers and migrant workers has become more prominent. This "semi-acquaintance" structure of rural society is changing. With the development of urbanization, the life style of some farmers who leave the countryside to the city not only reflects the change from "acquaintance" structure to "semi-acquaintance" structure, but also presents the trend of transformation to "stranger" structure $^{10}$.

On the other hand, college students returning home to start their own businesses and returning migrant workers have added a new atmosphere to the original rural areas. They have brought information from the outside world. And their language habits have changed to a certain extent, which exert a subtle influence on the language life of rural society. The language use and linguistic views of returnees affect the language use of local farmers. According to Wang Qing and Wu Xiaojun's survey ${ }^{8}$ of returning migrant workers in Sichuan Province, "the language variation of returning migrant workers shows obvious lexical characteristics, and the variation of dialects mainly takes the internal adjustment of dialects as the main means". Returning college students' understanding of the country's common language also affects the linguistic views of the original rural villagers ${ }^{7}$.

\section{The Influence of Mass Media on Rural Language Life}

According to some relevant reports, the coverage rate of broadcasting and television in China's rural areas reached $92.4 \%$ and $94.3 \%$ respectively in 2005 . More than $95 \%$ of the information obtained by farmers in China comes from radio and television. And the time for them to watch TV is about 2-3 hours per day. The number of rural netizens in China has reached 52.62 million, and mobile Internet access has gradually become a scale in rural areas, accounting for $23 \%$ of the total number of Internet users. By the end of 2012 , the Internet penetration rate of farmers had reached $23.7 \%$. And the proportion of new netizens from the countryside began to exceed that of cities and towns in 2012.

The emergence of telephone, television, radio and internet has changed the communication channels of rural information to a certain extent. The rural information exchange has gradually moved from "acquaintance" society to "stranger" society. In the form of information dissemination, it has broken through the face-to-face interpersonal communication. The "machine-human" one-

\footnotetext{
Chang Yuehua. Analyses and Considerations on the Status Quo of College Students' Diglossia Application: Standard Chinese and Dialects, Journal of Hainan Normal University, 2007(3).
} 
way information dissemination form with TV and radio as the information medium as well as the "human - machine - human" two-way information dissemination model formed by the Internet, QQ space, microblog and WeChat have gradually changed the language habits of rural people. People's modes of communication tend to be diversified. The language life of rural people has formed a two-line communication mode, with traditional rural acquaintance society as the main line of communication and new mass media such as the Internet as the auxiliary line of communication.

According to the Survey Report on the Internet Usage in Chinese Rural Areas, $37.3 \%$ of rural residents believe that they can not work or study without the Internet; $57.9 \%$ of rural residents believe that their lives will be monotonous without the Internet; $72.5 \%$ of the residents make new friends through the Internet; and $44.5 \%$ of the residents acknowledge that the Internet is the main channel for them to express their opinions. The emergence of virtual language life circles such as the Internet has become part of the language life of rural residents. And it is subtly changing the habits of rural language life.

\section{NEW GROUPS IN CURRENT RURAL LANGUAGE LIFE}

\section{A. Language Life and Psychological Loneliness of Empty Nesters}

According to the fifth national census, there are 11.179 million rural elderly "empty-nest" households, accounting for $71.58 \%$ of the total number of elderly "empty-nest" households. With the acceleration of urbanization and the migration of rural young and middle-aged workers, emptynest families in rural areas have shown a growing trend. Empty-nest household surveys in sociology and demography generally include three aspects: economic status and support, daily life care and spiritual comfort. Numerous studies have shown that the language use of empty nesters is a great part of their spiritual comfort ${ }^{8}$.

The traditional rural family structure is a three-generation family or a family of four generations. And there are many opportunities for face-to-face contact between family members, and the frequency of language use among family members is high. Because of the "acquaintance society" speech community in the village, there are many opportunities for communication between neighbors and the people in the village. With the first generation of rural migrant workers going out to work, the language use of empty nesters in rural areas is gradually narrowed and the opportunities for communication are reduced. Thus their psychological loneliness is higher. On the other hand, due to the limitation of information exchange channels, age, education and other factors, the rural empty nesters have a low rate of use and a low degree of acceptance of modern

Liao Heping \& Fu Rui. Analysis of the Main Problems and Causes of the Elderly of Rural Empty - Nest Family under the Background of Society Transition: Based on Survey Data from 18 Villages of 5 Provinces, Journal of Hunan University of Science \& Technology (Social Science Edition), 2012 (6). media, such as newspapers, television and the Internet. The survey by Li Jianxin, Feng Yingying and Yang Peng ${ }^{9}$ shows that the loneliness of empty nesters in rural areas is higher than that of non-empty nesters. And their living standard as well as life satisfaction is lower than that of non-empty nesters. Besides, the empty nesters are at a disadvantage in material living conditions, psychological status and subjective satisfaction.

To investigate the language life of empty nesters, we should first describe clearly the living conditions and language use of empty nesters in rural areas, such as the object of language use, the way of language use, the number and frequency of language use, daily communication content, etc. Secondly, the relationship between language life and psychological loneliness of empty nesters should be clarified. This can be done from the two dimensions of family and village community to investigate the density, complexity and degree of aggregation of their verbal communication in an attempt to explore the relationship between their language use and psychological loneliness. In other words, what is their family language use, the communication environment in the speech community, their attitude towards newspapers, television and the Internet? And what is the relationship between these language factors and the age, gender and personality of empty nesters? Furthermore, we should explore what kind of communication content, communication mode and communication strategy is more conducive to eliminating the loneliness of empty nesters, and thus improves the quality of life of empty nesters.

\section{B. Language Life and Language Skills of Left-behind Children}

According to the statistics, there are currently 61.125 million left-behind children in rural areas, accounting for $37.7 \%$ of all the rural children and $21.88 \%$ of the whole country's children. Because of their parents working in other places, there are problems in the left-behind children's lives, psychology and education. Most left-behind children are psychologically lonely because of the defects of fatherly love and maternal love. Relevant research also uses children's language communication as an indicator to measure the psychological loneliness of left-behind children ${ }^{10}$. At present, the relationship between left-behind children's language life and psychological loneliness is unclear.

Zhan Haiyu and Chen Shihai ${ }^{11}$ investigated the language communication between left-behind children in rural areas and their fathers working in cities. They found that there are low frequency of language communication between leftbehind children in rural areas and their parents who work in cities. In addition, their communication channels were single,

$9 \quad$ Li Jianxin \& Feng Yingying \&Yang Peng. Study on Quality of Life for the Elderly in Rural Empty Nests, Scientific Research on Aging, 2014(5).

10 Fan Xinghua \& Fang Xiaoyi \& Liu Qinxue \&Liu Yang. A Social Adaptation Comparison of Migrant Children, Rear Children, and Ordinary Children, Journal of Beijing Normal University (Social Sciences), 2009 (5).

11 Zhan Haiyu \& Chen Shihai. The Empirical Research on Daily Communication Between Migrant Workers and Their Left-home Children, Journal of Northwest A\&F University (Social Science Edition), 2012 (4). 
the content of their communication was monotonous, and the tendency of utilitarianism was heavy. On the level of communication technology, the understanding of the principles of language communication between migrant workers and left-behind children is relatively inadequate, and the ability of language communication is generally weak. Parents' neglect of the communicative context leads to their children's resentment. And misunderstanding of left-behind children's needs leads to the fact that migrant workers pay little attention to conversational strategies when communicating with their children. Moreover, more than half of the left-behind children live with their grandparents or other relatives, most of whom are poorly educated and more than half of whom graduate from primary school or have not attended school" ${ }^{12}$. Will there be a "language generation gap" when they communicate with children? Whether the communication topics between them and the left-behind children will be limited? And whether the education background of the elders will affect the language expression of the left-behind children?

In addition, left-behind children also face a period of entering the society and communicating with others. The current research is not enough for whether the language life of left-behind children has an impact on their language development and language communicative competence. Besides, the current research is not enough for finding out the impacts. Shi Aihua ${ }^{13}$ found that the linguistic competence of left-behind children in rural areas was significantly lower than that of non-left-behind children in terms of listening, observation, imagination, understanding and expression, which may be attributed to their limited language use environment, lack of parental love and lack of early childhood education. However, it cannot accurately reveal the relationship between the language life of left-behind children and their ability to use language. All these questions are worth studying.

Most of the left-behind children living in the countryside are cared for by their grandparents. Their language communication is a kind of "intergenerational communication". The language use and attitude of the caregivers directly affect children's language use. The leftbehind children's language use circle is a family language use circle formed by caregivers; a communication circle formed by children of the same age, and a school language use circle. How do the guardian's dialect background and the language use of children of the same age group affect their language use? "For children whose mothers work outside, their school language and family language tend to use dialects. In school language, women tend to use Mandarin than men."14 "The stronger the dialect habits of guardians, the more inclined children are to use dialects. When parents

12 Duan Chengrong \& Yangge. Study on the Latest Situation of Floating Children in China, Population Journal, 2008 (6).

13 Shi Aihua. The Deficiency of Preschool Education of Leftbehind Children: the test of and reflection on "the preschool learning capacity" of 516 children aged 6, Studies in Preschool Education, 2012 (4)

Fu Gan. Influence of Parents' Migration on Children's Language Use in the Rural Area: A Case Study of Funing County of Jiangsu Province, Applied Linguistics, 2016 (2) ask guardians to use Mandarin to take care of their children, the role of guardians' dialectal habits in the use of children's dialects will be weakened." 15 Therefore, for the language teaching of left-behind children in rural areas, we should pay attention to the influence of intergenerational communication on their language use, and put forward possible strategies, such as strengthening the communication between parents and left-behind children, requiring guardians to use Mandarin, etc.

Language is not only a tool for communication, but also a tool for identification. And it is accompanied by a language dividend. As a special group, left-behind children in rural areas should be paid more attention in their family language planning. A top-level design should be given to them as soon as possible in order to cultivate their multi-lingual ability.

\section{Returning Migrant Workers, Migrant Children and Language Adaptation, Linguistic Variation}

Migrant workers working in cities have the problems of language adaptation and language attitude transformation under the new environment. After returning home, they have brought a new perspective of dialect research, that is dialect variation. $\mathrm{Fu}$ Yirong ${ }^{7}$, Wang Qing and $\mathrm{Wu}$ Xiaojun's surveys8 of migrant workers returning home show that rural residents still use their native dialect after returning home, but their language use has undergone certain variations in phonetics, vocabulary, grammar and other aspects. Then, will the length of time for returning migrant workers to work in cities and the types of jobs have the effects on the language use after they return home? What's the change of their language attitude and language life after returning home? What is the relationship and differences between their language use in their hometown and city? These should be the contents of language life survey.

We have such examples around us: some children whose parents are migrant workers are born in the city and they live with their parents while their residences remain in the countryside. At a certain age of schooling, these children are sent back to the countryside to study and they live with their grandfather, grandmother or other caregivers. These migrant children may have learned to speak Mandarin fluently in the city. Then what about their language use when they interact with the children from the same village in their hometown? What about the communication between caregivers and migrant children? Are there any adjustments and changes in their language communication strategies? Are these adjustments and changes worth exploring?

To sum up, the three groups of rural language life show certain characteristics in their language life. Empty nesters and left-behind children mostly have the problem of language output in the monolingual environment. That is, their language use domain is narrow; their scope of use is limited; and their communication mode is single. The investigation of the language life of empty nesters and leftbehind children should focus on the number, frequency,

15 Li Jinfeng \& He Hongfeng \&Zhou Yuliang. Language Attitude, Language Environment and Language Use of Preschool Left-Behind Children in Rural Areas, Applied Linguistics, 2017 (1) 
objects, occasions, and methods of their language use. Besides, the investigators should understand these groups' language use and strategies in different situations and explore the relationship between language life, language expression ability and psychological loneliness. We can use the concept of "quality of life" for reference and try to establish a "language quality of life" system to measure the language use of specific groups in language life. To investigate the language life of migrant workers returning home, we should focus on their language adaptation and linguistic variation.

\section{RESEARCH ON PRIMARY ORALITY}

The word "primary orality" first appeared in Walter J. Ong's concept of "primary oral culture ${ }^{16 \%}$. In Walter J. Ong's words, "...the orality of a culture totally untouched by any knowledge of writing or print, 'primary orality'. It is 'primary' by contrast with the 'secondary orality' of presentday high-technology culture, in which a new orality is sustained by telephone, radio, television, and other electronic devices that depend for their existence and functioning on writing and print..." On the basis of this definition, we hold that primary orality is "spoken language acquired naturally by people without language education in the context of oral communication. ${ }^{17,}$ " On the one hand, the language hosts of primary orality do not have access to language education, and do not understand the logical way of thinking in written language. What they use is a "limited code". On the other hand, primary orality remains "untouched by any knowledge of writing". Thus it is not the voiced form of written language.

According to relevant data, the population of the whole country was 550 million in 1949 , of which more than 400 million were illiterate. The illiteracy rate was as high as $80 \%$, and in rural areas the illiteracy rate was more than $95 \%$. In 2005, there were 85 million illiterates in China, of which $4.8 \%$ were young and middle-aged illiterates aged 15-50, and $90 \%$ of these illiterates were distributed in rural areas. On the one hand, the countryside is the most concentrated area of illiteracy. On the other hand, the rural area is a closed acquaintance society based on blood relationship and geographical relationship in the traditional sense. The living radius of the farmers is limited; the language blends and variations are small. The language used by the illiterates in the rural areas can best represent the original features of the local language. In John J. Gumperz's opinion ${ }^{18}$, the characteristics of closed network relationships result in the characteristics of communication activities among its members. The exclusive characteristics and the common cultural conventions between network members restrict the use of communication codes among network members. The norms restricted by context make the conversations within

\footnotetext{
16 Walter J.Ong., Orality and Literacy: The Technologizing of the word, Translated by He Daokuan, Peking University press, 2008.

17 Guo Qijun. Research on Primary orality in Qin'an district of Gansu province, The Doctor's degree of Jinan University, 2017.

18 John J. Gumperz. Discourse strategies, Translated by Xu Daming \& Gao haiyang, Social Sciences Academic Press 2001.
}

closed network groups more and more stylized. In this tacit understanding, communication becomes simple and efficient.

At present, there is a growing demand for the investigation of endangered languages and dialects. The basic paradigm is to follow the American descriptive school's practice of collecting corpus by reading word lists, vocabulary lists and sentences, and then sum up the phonetic, lexical and syntactic features of the local language. However, natural conversation and discourse materials are few. In the study of endangered languages and dialects from the perspective of primary orality, more attention should be paid to the recording of natural conversational corpus in paragraphs, so as to show the true and multi-angle local original language style. Therefore, under the current historical conditions, the investigation and study of primary orality is not only a record of the linguistic features of the illiterate group, but also the preservation of the original local languages and dialects. It is also a description of the linguistic features of the initial stage of human language development. By investigating primary orality, we can understand the language used by people in the "acquaintance society", which is a limited life circle. We can also understand the way of language communication in the context of oral communication, and record the original language and cultural features of a region. By comparing the spoken language of the illiterates with that of the educatee, we can understand the influence of language education on spoken language use.

For now, there are two main aspects in the investigation of rural primary orality: first, the description of primary orality, such as the description of basic vocabulary, common vocabulary, specific vocabulary, specific sentence pattern, statistics of average sentence length, description and ranking of common language functions; second, surveys of the language life of these groups, such as the objects they usually associate with, the topics they communicate with each other, the occasions and ways of communication. All these can be studied to explore the impact of language life on these groups' language use. At present, with the improvement of the level of education for all and the impact of mass media, there will be fewer and fewer primary orality in the countryside. Thus the study of primary orality becomes more and more important.

\section{CONCLUSION}

Compared with the urban language survey, there is no unified paradigm in the theoretical and methodological aspects of rural language survey, and the research results are rare. In the new period of rural language survey, we should adapt to the new changes in rural language life, pay attention to the special groups in rural language life, and display their language life in an all-round way. This will help us understand the language life of specific groups in rural areas, and explore the relationship between language life, psychological loneliness and language expression ability. It is of practical significance to solve the difficulties in language life of such specific groups. As a language used in the context of oral communication, primary orality has practical and theoretical significance for us to understand the 
mode of language communication in the context of oral communication, to preserve the original language culture in rural areas, and to explore the impact of language education on language use. Therefore, we should learn from the theories and achievements of the urban language survey and go deep into the rural language survey.

\section{REFERENCES}

[1] Chen Weilin, Chen Xingyan. The Investigation and Reflection on the Situationof Tuipu in the Rural Primary and Middle Schools, Applied Linguistics, 2004(4).

[2] Zhao Xiaogang. A Study on the Perception of General Language and Writing of People in Rural Gansu, Applied Linguistics, 2007(4).

[3] FU Yirong. Migration and the Popularization of Putonghua:A Case Study of Migrant Workers from Fucun Village of Wuwei, Anhui Province, Applied Linguistics, 2010(2).

[4] WANG Qing \& Wu Xiaojun. New Research Perspective of Common Language and Dialect Variation - An Empirical Study on Pronunciation Variations of Sichuan Returned Migrant Workers, Journal of Xihua University (Philosophy \& Social Sciences), 2012(6).

[5] Fei Xiaotong: " From the Soil :The Foundations of Chinese Society ", Peking University press, 2005.

[6] Li Hongyan. Rural Communication Studies, Peking University press, 2014.

[7] Chang Yuehua. Analyses and Considerations on the Status Quo of College Students' Diglossia Application: Standard Chinese and Dialects, Journal of Hainan Normal University, 2007(3).

[8] Liao Heping \& Fu Rui. Analysis of the Main Problems and Causes of the Elderly of Rural Empty - Nest Family under the Background of Society Transition: Based on Survey Data from 18 Villages of 5 Provinces, Journal of Hunan University of Science \& Technology (Social Science Edition), 2012(6).

[9] Li Jianxin \& Feng Yingying \&Yang Peng. Study on Quality of Life for the Elderly in Rural Empty Nests, Scientific Research on Aging, 2014(5).

[10] Fan Xinghua \& Fang Xiaoyi \& Liu Qinxue \&Liu Yang. A Social Adaptation Comparison of Migrant Children, Rear Children, and Ordinary Children, Journal of Beijing Normal University(Social Sciences), 2009(5).

[11] Zhan Haiyu \& Chen Shihai. The Empirical Research on Daily Communication Between Migrant Workers and Their Left-home Children, Journal of Northwest A\&F University(Social Science Edition), 2012(4).

[12] Duan Chengrong \& Yangge.Study on the Latest Situation of Floating Children in China, Population Journal, 2008(6).

[13] Shi Aihua. The Deficiency of Preschool Education of Left-behind Children :the test of and reflection on " the preschool learning capacity" of 516 children aged 6, Studies in Preschool Education, 2012(4).

[14] Fu Gan. Influence of Parents' Migration on Children's Language Use in the Rural Area: A Case Study of Funing County of Jiangsu Province, Applied Linguistics, 2016(2).

[15] Li Jinfeng \& He Hongfeng \&Zhou Yuliang. Language Attitude, Language Environment and Language Use of Preschool Left-Behind Children in Rural Areas, Applied Linguistics, 2017(1).

[16] Walter J.Ong., Orality and Literacy: The Technologizing of the word ,Translated by He Daokuan, Peking University press, 2008.

[17] Guo Qijun. Research on Primary orality in Qin'an district of Gansu province, The Doctor's degree of Jinan University, 2017.

[18] John J. Gumperz. Discourse strategies, Translated by Xu Daming \& Gao haiyang, Social Sciences Academic Press 2001. 